• 研究报告・

\title{
丰林典型阔叶红松林地表鞘翅目成虫空间异质性 及其与环境因子的空间关联性
}

\author{
倪娟平 ${ }^{1,2}$ 程赛赛 ${ }^{1,2}$ 高梅香 ${ }^{1,2^{*}}$ 卢廷玉 ${ }^{1,2}$ 金光泽 $^{3}$ \\ 1 (哈尔滨师范大学地理科学学院, 哈尔滨 150025) \\ 2 (黑龙江省普通高等学校地理环境遥感监测重点实验室, 哈尔滨 150025) \\ 3 (东北林业大学生态研究中心, 哈尔滨 150040)
}

\begin{abstract}
摘要: 土壤动物群落空间异质性及其与环境因子的空间作用关系, 是揭示土壤生态系统格局与过程及生物多样性 维持机制的重要基础。作者于2015年生长季节(8月)、寒冷季节(10月)在丰林典型阔叶红松林动态监测样地内, 采 用陷阱法调查地表鞘翅目成虫群落, 基于地统计空间分析方法, 揭示步甲科和隐翅虫科群落个体数和物种数及优 势种的空间格局, 并分析这些空间格局与土壤含水量和地形因子的空间关联性。两次采样共捕获步甲科成虫26种 617 只, 隐翅虫科19种222只。8月群落个体数和物种数表现为中等变异, 10月为强变异, 群落组成在两个月间具有 显著差异。生长季节(8月)和寒冷季节(10月)步甲科和隐翅虫科群落多表现为中等的空间自相关性, 空间分异由随 机性因素和结构性因素共同决定。单个物种的个体数多具有中等的空间异质性特征, 且其空间分异主要由随机性 因素和结构性因素共同调控。生长季节群落的个体数、物种数和优势种个体数多形成斑块和孔隙镶嵌分布的空间 格局。物种之间及物种与环境因子之间多为复杂的空间关联性, 这些关联性主要受到结构性因素或随机性因素的 单一调控。典范对应分析(canonical correspondence analysis, CCA)结果表明, 8月土壤含水量对步甲科和隐翅虫科物 种分布影响显著, 10月凹凸度对步甲科分布影响显著, 海拔对隐翅虫科分布具有显著影响。本研究表明地表步甲科 和隐翅虫科在生长季节形成明显的空间格局而在寒冷季节空间格局不明显, 为不同尺度地表土壤动物空间异质性 和生物多样性维持机制研究提供了理论基础。
\end{abstract}

关键词: 步甲科; 隐翅虫科; 地形因子; 空间异质性; 空间关联性; 阔叶红松林

\section{Spatial heterogeneities of ground-dwelling Coleoptera adults and their spatial correlations with environmental factors in a typical broad-leaved Korean pine forest in the Fenglin Nature Reserve}

\author{
Juanping $\mathrm{Ni}^{1,2}$, Saisai Cheng ${ }^{1,2}$, Meixiang Gao ${ }^{1,2^{*}}$, Tingyu $\mathrm{Lu}^{1,2}$, Guangze $\mathrm{Jin}^{3}$ \\ 1 College of Geographical Sciences, Harbin Normal University, Harbin 150025 \\ 2 Key Laboratory of Remote Sensing Monitoring of Geographic Environment, College of Heilongjiang Province, Harbin \\ Normal University, Harbin 150025 \\ 3 Center for Ecological Research, Northeast Forestry University, Harbin 150040
}

\begin{abstract}
Spatial heterogeneities of soil animal communities and their associations with environmental factors are important for revealing the patterns and processes of soil ecosystems and maintenance mechanisms of soil biodiversity. This experiment was conducted in a typical mixed broad-leaved Korean pine (Pinus koraiensis) forest plot in the Fenglin Nature Reserve in August and October of 2015. Geostatistics was used to reveal the spatial patterns of species number and individuals of ground-dwelling Carabidae and Staphylinidae adult communities and dominant species, and to explain the associations between these spatial patterns and soil water content and topographic variables. In total, 26 and 19 species of Carabidae and
\end{abstract}


Staphylinidae beetles were caught and 617 and 222 individuals were collected, respectively. Variabilities in individuals and species numbers of communities were moderate in August and strong in October. Community compositions were significantly different between the two months. Carabidae and Staphylinidae communities showed moderate spatial autocorrelations in both growing (August) and relatively cold (October) seasons. Spatial heterogeneities of the Carabidae and Staphylinidae communities were determined by both random and structural factors. However, most species individuals exhibited significant spatial heterogeneities and these heterogeneities were controlled by structural factors. Individuals and species number of communities and dominant species individuals formed mosaic patterns with patches and gaps. Spatial associations between individuals and species numbers of communities and dominant species individuals with environmental factors were complex. Spatial associations were mainly controlled by structural or random factors. CCA analysis showed that soil water content in August had a significant effect on the species distribution of Carabidae and Staphylinidae adults in August. In October, the convexity had a significant impact on the distribution of Carabidae adults, and altitude was significantly related to the distribution of Staphylinidae adults. This experiment suggests that the spatial heterogeneities of Carabidae and Staphylinidae adults were obvious in the growing season, but not obvious in the relatively cold season. The results of this study will help us to understand the spatial variation and biodiversity maintenance mechanisms of soil animal communities at multiple scales.

Key words: Carabidae; Staphylinidae; topographic factors; spatial heterogeneity; spatial association; mixed broad-leaved Korean pine forest

空间异质性是生态系统在空间上的复杂性和 变异性(Li \& Reynolds, 1995), 是群落空间格局形成 的基础，也是物种共存的重要前提。空间异质性越 高, 意味着有更多的小生境、更多样的小气候条件 以及更多的躲避捕食的隐蔽所，因而能允许更多的 物种共存。空间异质性不仅在促进捕食者和被捕食 者的物种共存中发挥着潜在作用(Holt，1984), 异质 性的土壤质地还有利于增强物种对环境变化的适 应能力(Fernandez-Going, 2014)。草地生态系统中, 物种空间异质性改变后(增加新的植被物种)对物种 维度的增加和原有物种的组成都有重要影响 (Questad \& Foster, 2008)。因此, 深入挖掘空间异质 性的性质、成因和潜在的生态学效应, 有利于从空 间格局的角度更好地揭示生态学过程背后的成因 机制(高梅香等, 2016)。

作为生态系统重要的分解者和消费者, 土壤动 物在维持生物多样性和生态系统功能方面具有重 要作用, 在多种时空尺度上均表现出明显的异质性 特征(Gutiérrez-López et al, 2010)。很多环境条件如 植被类型、土壤结构、土壤含水量、土壤化学性质、 土壤温湿度等(Sousa et al, 2003)都可能对土壤动物 异质性格局产生影响。我国土壤动物空间异质性的 研究开展较晚, 目前主要集中于较小的空间尺度 (Li \& Reynolds, 1995), 对土壤动物在各种不同生态 系统中的空间分异规律研究相对较少(尹文英,
1997), 需要开展多尺度条件下的空间异质性研究。

阔叶红松(Pinus koraiensis)林结构复杂、组成独 特、生物多样性丰富, 是东北典型的温带针阔叶混 交林地带性顶极植被, 是北温带生物多样性最高的 森林类型(李俊清和李景文, 2003)。地表鞘翅目昆虫 在生态系统中扮演着重要的角色, 在维持生物多样 性和生态系统功能方面具有重要作用(周红章等, 2014), 是解释多尺度空间地表生态系统格局与过 程的良好实验对象(Rainio \& Niemelä, 2003)。阔叶 红松林内地表鞘翅目昆虫物种丰富, 形态特殊、食 性行为多样、适应性广且数量大。因此本实验以丰 林典型阔叶红松林为研究地区, 在东北林业大学 2005年建立的阔叶红松林固定监测样地内, 于2015 年8、10月采用陷阱法捕获地表鞘翅目成虫, 基于地 统计空间分析方法研究地表步甲科和隐翅虫科群 落的物种组成、空间异质性和空间关联性，分析不 同群落和物种个体数与地形因子的空间关联性, 以 期为土壤动物群落构建机制研究奠定基础。

\section{1 研究区概况}

研究区位于黑龙江省丰林国家级自然保护区 内 $\left(128^{\circ} 59^{\prime}-129^{\circ} 15^{\prime} \mathrm{E}, 48^{\circ} 02^{\prime}-48^{\circ} 12^{\prime} \mathrm{N}\right)$, 该区是目 前世界上保存最为完整的为数不多的北温带原始 阔叶红松林生态系统之一(刘林馨等, 2011)。研究区 为低地丘陵, 土壤以山地棕色森林土为主。属于 
大陆性季风气候, 年均温 $-0.5^{\circ} \mathrm{C}$, 年平均降水量 $640.5 \mathrm{~mm}$ 。地带性植被类型是以红松为优势种的针 阔混交林。

\section{2 研究方法}

\section{1 样地设置与数据采集}

实验样地设置在东北林业大学于2009年在丰 林自然保护区建立的阔叶红松林30 ha固定监测样 地内, 以该固定监测样地西北角 9 ha范围作为实验 样地, 以 $20 \mathrm{~m}$ 为间隔将样地划分为 225 个 $20 \mathrm{~m} \times$ $20 \mathrm{~m}$ 的单元格, 共计 256 个网格交叉点。于 2015 年 8 月和 10 月在以网格交叉点(交叉点置有水泥桩)为圆 心的 $20 \mathrm{~cm}$ 半径圆范围内通过陷阱法采集样品(每次 256 个采样点), 当该范围内存在积水、树桩、倒木、 大块岩石时，选择在该网格交叉点 $50 \mathrm{~cm}$ 半径圆内 布置陷阱。先用内径 $7 \mathrm{~cm}$ 的土钻挖取一个 $15 \mathrm{~cm}$ 深 的柱状土坑, 将高 $14 \mathrm{~cm}$ 、内径 $7 \mathrm{~cm}$ 的塑料诱捕杯置 于坑内, 并使杯口与地面齐平。诱捕杯中加入约占 $2 / 3$ 容积的饱和 $\mathrm{NaCl}$ 溶液, 同时在杯口上部距离地 面约 $10 \mathrm{~cm}$ 处支起 1 个一次性餐盘作为防护, 陷阱置 于野外7天7夜后取回。在陷阱附近用铝盒在约 $7 \mathrm{~cm}$ 深处采集土样, 采用烘干法, 依据(土壤湿重 - 土 壤干重)/土壤干重 $\times 100 \%$ 计算土壤含水量。

地形因子数据为东北林业大学依照BCI (Barro Colorado Island) 50 ha样地的技术规范, 将监测样 地设置成 $10 \mathrm{~m} \times 10 \mathrm{~m}$ 的样方, 调查的每个样方的地 形因子数据, 包括海拔、坡度、坡向和凹凸度。地 形因子的测量方法参见 Gutiérrez-López 等(2010), 将每个 $20 \mathrm{~m} \times 20 \mathrm{~m}$ 样方内 4 个 $10 \mathrm{~m} \times 10 \mathrm{~m}$ 样方的均 值作为该样方的地形因子数据。

室内将捕获到的土壤动物样品挑拣出来, 置于 95\%医用酒精中保存。参照《原色中国东北土壤甲 虫图鉴: 步行虫类》 (李井科等, 2015a) 和《原色中国 东北土壤甲虫图鉴: 隐翅虫类, 拟步甲类》 (李井科 等, 2015b) 将鞘翅目昆虫鉴定到种，鞘翅目成虫与 幼虫分别计数。由于幼虫难以鉴定到种, 因此本文 仅将步甲科和隐翅虫科成虫用于后续分析处理。

\section{2 数据分析}

\subsection{1 鞘翅目成虫群落基本特征}

以每个陷阱获取的数据作为 1 个样本, 计算鞘 翅目成虫个体数、标准差和变异系数 (coefficient of variation, $C V)$ 。变异系数 $(C V)=$ 标准差/平均值,
$C V<0.1$ 时, 为弱变异, $0.1 \leq C V \leq 1$ 时为中等变异, $C V>1$ 时为强变异(雷志栋等, 1988)。同时对鞘翅目 成虫群落个体数和物种数进行正态分布检验，对不 符合正态分布的数据进行 $\ln (x+1)$ 转换, 使之符合 或近似符合正态分布, 进行地统计学分析。利用独 立样本 $t$ 检验来考察 8 月和 10 月间步甲科和隐翅虫科 个体数及物种数的差异显著性。

\subsection{2 半方差分析}

采用半方差分析(semi-variance analysis)探讨步 甲科和隐翅虫科群落及优势种的空间异质性特征, 半方差函数的计算公式为:

$$
\gamma(h)=\frac{1}{2 N(h)} \sum_{i=1}^{N(h)}\left[\left(Z\left(x_{i}\right)-Z\left(x_{i}+h\right)\right)\right]^{2}
$$

式中, $\gamma(h)$ 为变异函数, 揭示了整个尺度上的空间变 异。 $h$ 是样本间距, 又称滞后距离, $N(h)$ 为距离为 $h$ 时 对应的样点对数, $Z\left(x_{i}\right)$ 和 $Z\left(x_{i}+h\right)$ 分别为区域化变量 $Z$ 在空间位置 $x_{i}$ 和 $x_{i}+h$ 处的观测值。

计算不同距离的变异函数值, 拟合出半方差函 数模型, 根据模型参数定量描述变量的空间异质性: (1)块金值 $\left(C_{0}\right)$ 代表由实验误差和小于最小取样尺度 引起的随机部分的空间异质性(吴青柏等, 2003), 其 值大表明较小尺度上的某种过程不可忽视; (2)结构 方差 $(C)$ 表示由于土壤母质、地形、气候等非人为的 区域因素引起的变异; (3) $\left(C_{0}+C\right)$ 阈值(基台值Sill) 是变量在研究范围内最大的变异程度, 其值越大表 示总的空间变异性程度越高; (4)结构比 $C /\left(C_{0}+C\right)$ 表示自相关部分引起的空间变异程度的高低, 它反 映了空间相关性的程度和结构性因素引起的空间 异质性占总变异的比例, 结构比 $C /\left(C_{0}+C\right)>0.75$ 、 $0.25-0.75$ 、 $<0.25$ 分别表示变量空间自相关性较强 (空间变异主要由结构性因素引起)、中等(空间变异 由随机性因素和结构性因素共同决定)和很弱(随机 部分引起的空间变异起主要作用)(Cambardella et al, 1994); (5)变程 $\left(A_{0}\right)$ 表示区域化变量存在空间自相关 特性的平均最大距离(郭旭东等, 2000), 是野外研究 选样尺度的理论依据(李元寿等, 2008)。

\subsection{3 交叉方差函数分析}

交叉方差函数(cross-variogram)用于分析鞘翅 目成虫物种之间、物种与环境因子之间的空间作用 关系以及相互作用的范围大小(王正军等, 2002), 其 
公式为(Rossi et al, 1995):

$\gamma_{A B}(h)=\frac{1}{2 N(h)} \sum_{i=1}^{N(h)}\left[Z_{A}\left(x_{i}\right)-Z_{A}\left(x_{i}+h\right)\right]\left[Z_{B}\left(x_{i}\right)-Z_{B}\left(x_{i}+h\right)\right]$

式中, $\gamma_{A B}(h)$ 为相距为 $h$ 的变量 $A$ 与变量 $B$ 之间的交叉 方差函数值; $N(h)$ 为相隔距离为 $h$ 的所有点的配对数; $Z_{A}\left(x_{i}\right) 、 Z_{A}\left(x_{i}+h\right)$ 分别是变量 $A$ 在 $x_{i}$ 和 $\left(x_{i}+h\right)$ 处的观测 值; $Z_{B}\left(x_{i}\right) 、 Z_{B}\left(x_{i}+h\right)$ 分别是变量 $B$ 在 $x_{i}$ 和 $\left(x_{i}+h\right)$ 处的 观测值。最后利用简单Mantel检验( simple Mantel test)进一步检验鞘翅目成虫物种之间以及物种与环 境因子之间空间关系的显著性(Gutiérrez-López et al, 2010)。

\subsection{4 典范对应分析}

采用典范对应分析 (canonical correspondence analysis, CCA) 阐明步甲科和隐翅虫科成虫个体数 与含水量和地形因子的相关性。

\subsection{5 普通克里格空间插值}

根据Bivand等(2013)提出的插值方法决定树, 选择普通克里格对样地土壤动物个体数及环境因 子进行空间分布模拟。普通克里格(ordinary kriging) 插值是在有限区域内对区域化变量进行的无偏最 优估计(王政权, 1999), 其实质是一个实行局部估计 的加权平均值:

$$
Z\left(x_{0}\right)=\sum_{i=1}^{n} \lambda Z\left(x_{i}\right)
$$

式中, $Z\left(x_{0}\right)$ 是在未经观测的点 $x_{0}$ 上的内插估计值, $Z\left(x_{i}\right)$ 是在点 $x_{0}$ 附近的若干观测点上获得的实测值。

\subsection{6 交叉验证}

交叉验证(cross-validation) 分析是从已知数据 集中删除 1 个采样点, 用剩下的采样点估算删除点 的数值, 并计算误差均值绝对值和误差均方根。本 文对所获得的半方差函数模型进行交叉验证。符合 以下标准的方法为最优(贾艳红等, 2008; Xia et al, 2016): 平均预测误差(mean error, ME)最接近于 0 , 均方根误差(root mean square error, RMSE)最小。

在Microsoft Excel 2010中对原始数据进行基本 的分析和预处理。直方图正态分布检验与独立样本 $t$ 检验分析在SPSS 21.0中完成。交叉方差函数分析 在软件 $\mathrm{GS}+9.0$ 中进行, 半方差函数分析及其交叉验 证及 Mantel 检验通过 R3.4.3 软件中的 “geoR” 和 “vegan”软件包完成, 空间插值在 R3.4.3 软件中的 “gstat”包中实现。

\section{3 结果}

\section{1 鞘翅目成虫群落的统计描述}

2015 年 8 月捕获步甲科成虫 24 种 586 只，隐翅虫 科13种 207 只；10月捕获步甲科成虫 10 种 31 只，隐翅 虫科 10 种 15 只。 8 月捕获的物种数和个体数极显著 多于10月 $(P<0.01)$, 步甲科和隐翅虫科的群落组成 具有明显的时间变化特征。两个群落的个体数和物 种数 8 月均为中等变异, 10 月均为强变异。调查月份 的步甲科和隐翅虫科成虫个体数均不符合正态分布, 采用 $\ln (x+1)$ 转换使之符合或近似符合正态分布。

\section{2 半方差函数特征分析}

\subsection{1 步甲科和隐翅虫科群落的半方差特征}

交叉检验表明，步甲科的群落个体数 8 月拟合 为球状模型 (ME最接近于 0$)$, 结构比为 0.14 , 不具有 统计学意义上显著的空间结构; 10 月拟合为高斯模 型，结构比介于 0.25-0.75之间，具有中等空间自相 关性，由随机因素和结构性因素共同决定群落的空 间分异。隐翅虫科的群落个体数在 8 月拟合为高斯 模型，表现为中等的空间自相关性；10月拟合为球 状模型, 结构比为 1 , 具有较强的空间分异, 且由结 构性因素引起。两个群落个体数在两个月份的空间 自相关距离较大, 变程均大于 $100 \mathrm{~m}$ (图1)。

步甲科 $8 、 10$ 月的群落物种数均可用高斯模型 进行拟合，且两者结构比均在 0.25-0.75之间，说明 步甲科物种数具有中等的空间自相关性且空间分 异由随机因素和结构性因素共同决定。隐翅虫科8 月、10月的物种数分别可用高斯模型、球状模型进 行拟合, 分别表现为中等和较强的空间自相关性。 物种数的空间自相关性范围较大(图2)。

\subsection{2 步甲科和隐翅虫科物种的半方差特征}

本文选取个体数大于 $10 \%$ (优势种)或物种在样 地中出现的频率大于 $10 \%$ 的物种 $(8$ 月 6 个物种，10月 2 个物种)进行半方差函数分析。8月所分析的物种中 仅金边步甲(Megodontus vietinghoffi)的结构比大于 0.75 , 物种的空间变异主要由结构性因素引起, 且 其变程最大 $(450 \mathrm{~m})$; 其他物种的结构比均在 0.25-0.75之间, 空间变异由随机性因素和结构性因 素共同调控。10月2 个物种分别拟合为指数模型和 球状模型, 结构比均大于 0.75 。群落中所有物种的 块金值均大于 0 , 说明物种个体数的半方差中均存 

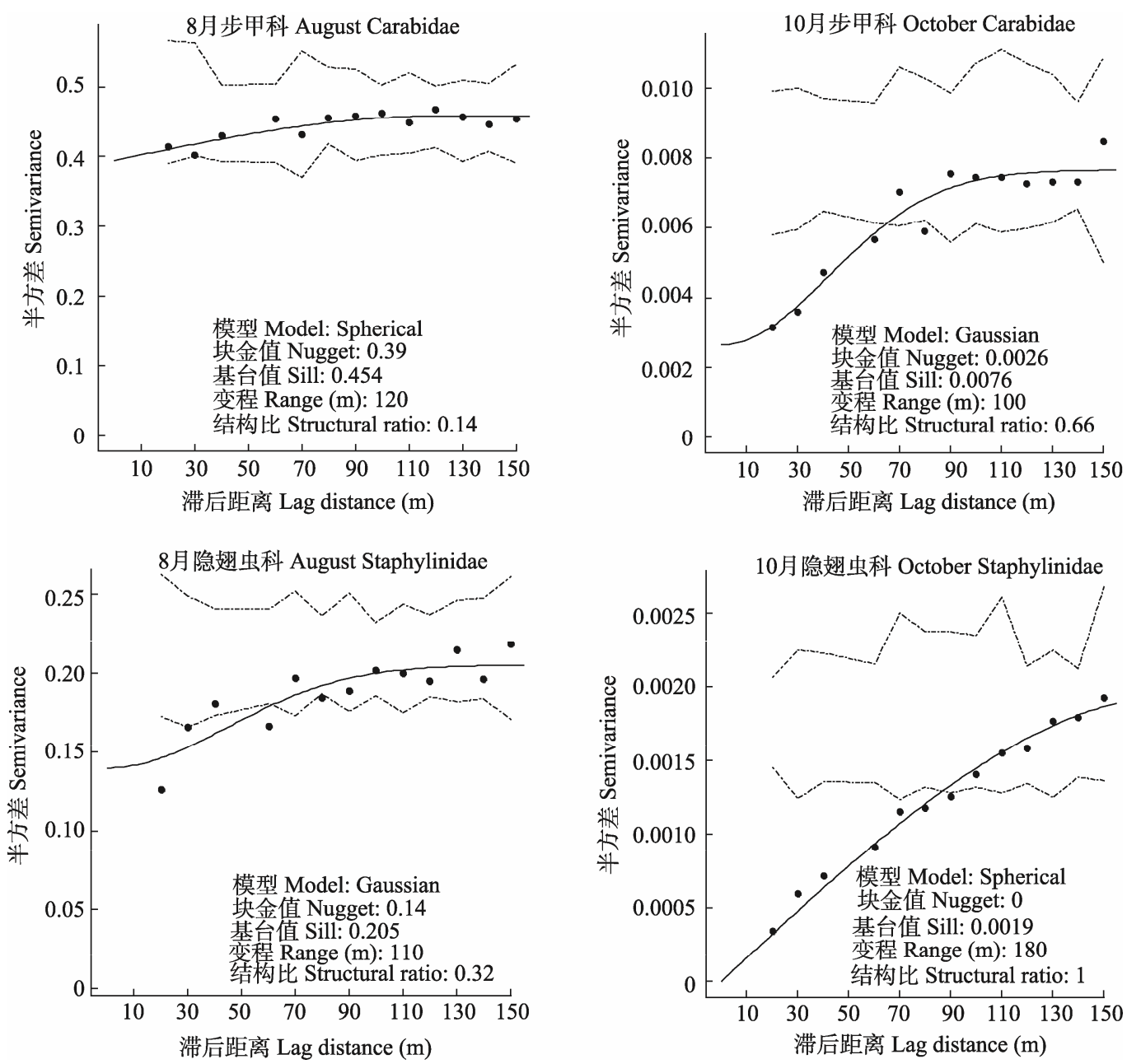

图1 步甲科和隐翅虫科成虫群落个体数半方差函数图。Spherical, Gaussian分别表示球状模型和高斯模型。两条虚线表示数 据显示为完全随机时的 $95 \%$ 置信区间, 实线为拟合的半方差函数曲线。

Fig. 1 Semivariograms of individuals for ground Carabidae and Staphylinidae adult communities. Spherical, Gaussian represent the spherical and Gaussian models. Two dashed lines represent the $95 \%$ confidence intervals when the data is completely random, and the solid line is the fitting half variance function curve.

在块金方差, 即存在由采样误差微尺度空间变异等 因素带来的块金效应(表1)。

\subsection{3 土壤含水量及地形因子的半方差函数特征}

8 月和 10 月样地土壤含水量具有显著差异 $(P<$ $0.05)$, 均可用高斯模型进行拟合, 结构比小于 0.25 , 含水量的空间分异主要由随机性因素调控。样地海 拔、凹凸度拟合为球状模型，坡度、坡向拟合为高 斯模型，地形因子的结构比大于 0.75 , 表明空间自 相关性较强, 其空间异质性主要由结构性部分引起 (表2)。

\section{3 空间插值分析}

\subsection{1 步甲科、隐翅虫科成虫群落个体数和物种数}

8 月步甲科、隐翅虫科的个体数和物种数的空 间分布格局相似，高值区集中在样地左上角和样地
的右侧边缘, 低值区在样地的中心位置。斑块等级 个体数略高于物种数, 步甲科群落略高于隐翅虫 科。步甲科的最小值出现在样地中心，隐翅虫科的 出现在样地上侧中心位置(图3)。10月份步甲科和隐 翅虫科成虫的个体数均较少, 未进行空间插值。

\subsection{2 步甲科、隐翅虫科成虫优势种个体数}

8月份步甲科的优势种Pterostichus adstrictus和 沟步甲(Aulonocarabus canaliculatus)及隐翅虫科的 优势种Philonthus wuesthoffi相似，均呈斑块状分布， 形成高值斑块和低值斑块镶嵌分布的格局, 样地中 心物种分布普遍较少，样地边缘较为集中(图4)。

\section{4 空间关联性分析}

\subsection{1 步甲科和隐翅虫科的物种间空间关联性}

8 月份物种间空间关联性结果表明，金边步甲 

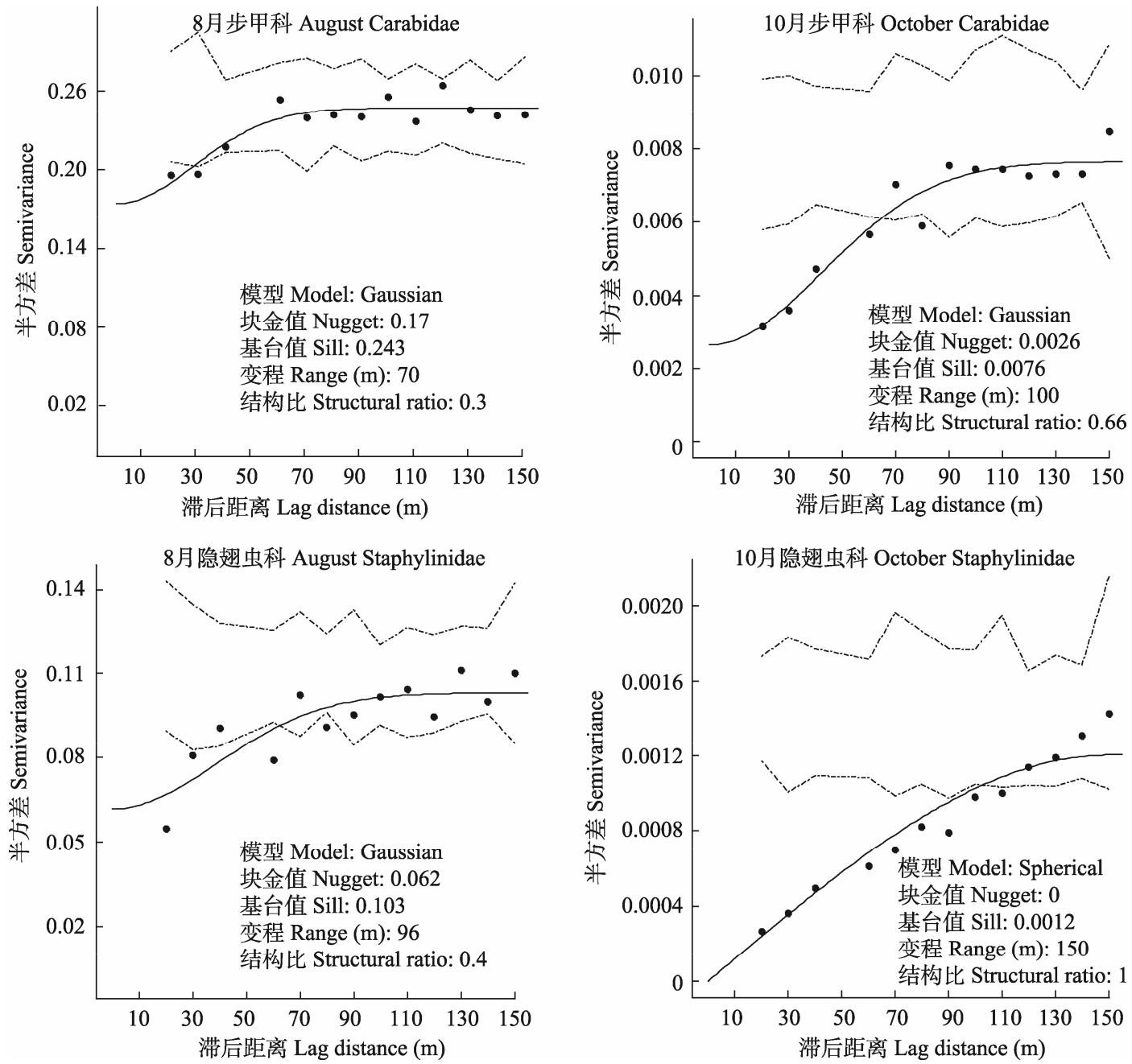

图2 步甲科和隐翅虫科成虫群落物种数半方差函数图。Spherical, Gaussian分别表示球状模型和高斯模型。两条虚线表示数 据显示为完全随机时的 $95 \%$ 置信区间，实线为拟合的半方差函数曲线。

Fig. 2 Semivariograms of sepcies number for Carabidae and Staphylinidae adult communities. Spherical, Gaussian represent the spherical and Gaussian models. Two dashed lines represent the $95 \%$ confidence intervals when the data is completely random, and the solid line is the fitting half variance function curve.

表1 物种的半方差函数理论模型和空间异质性参数

Table 1 Theoretical models and corresponding parameters for semivariograms of species individuals

\begin{tabular}{|c|c|c|c|c|c|c|}
\hline & $\begin{array}{l}\text { 物种 } \\
\text { Species }\end{array}$ & $\begin{array}{l}\text { 模型 } \\
\text { Model }\end{array}$ & $\begin{array}{l}\text { 块金值 } \\
\text { Nugget }\left(C_{0}\right)\end{array}$ & $\begin{array}{l}\text { 基台值 } \\
\text { Sill }\left(C_{0}+C\right)\end{array}$ & $\begin{array}{l}\text { 变程 } \\
\text { Range } A_{0}(\mathrm{~m})\end{array}$ & $\begin{array}{l}\text { 结构比 Structural } \\
\text { ratio }\left[C /\left(C_{0}+C\right)\right]\end{array}$ \\
\hline \multirow{6}{*}{$\begin{array}{l}\text { 8月 } \\
\text { August } 2015\end{array}$} & Pterostichus maoershanensis & 高斯模型 Gau & 0.005 & 0.018 & 140 & 0.734 \\
\hline & Pterostichus adstrictus & 指数模型 $\operatorname{Exp}$ & 0.110 & 0.210 & 150 & 0.476 \\
\hline & Carabus billbergi & 高斯模型 Gau & 0.008 & 0.020 & 110 & 0.597 \\
\hline & Megodontus vietinghoffi & 指数模型 Exp & 0.007 & 0.325 & 450 & 0.800 \\
\hline & Aulonocarabus canaliculatus & 球状模型 Sph & 0.120 & 0.199 & 150 & 0.397 \\
\hline & Philonthus wuesthoffi & 指数模型 Exp & 0.062 & 0.162 & 130 & 0.617 \\
\hline \multirow{2}{*}{$\begin{array}{l}\text { 10月 } \\
\text { October } 2015\end{array}$} & Pterostichus adstrictus & 指数模型 Exp & 0.001 & 0.003 & 330 & 0.805 \\
\hline & Platynus ezoanus & 球状模型 Sph & 0.000 & 0.001 & 150 & 0.812 \\
\hline
\end{tabular}

Exp, Sph, Gau represent the exponential, spherical and Gaussian model, respectively. 
表2 不同环境因子半方差函数理论模型及相关参数

Table 2 Theoretical models and corresponding parameters for semivariograms of environmental factors

\begin{tabular}{llllll}
\hline & 模型 & $\begin{array}{l}\text { 块金值 } \\
\text { Model }\end{array}$ & $\begin{array}{l}\text { 基台值 } \\
\text { Sigget }\left(C_{0}\right)\end{array}$ & $\begin{array}{l}\text { 变程 } \\
\text { Range } A_{0}(\mathrm{~m})\end{array}$ & $\begin{array}{l}\text { 结构比 } \\
\text { Structural ratio }\left[C /\left(C_{0}+C\right)\right]\end{array}$ \\
\hline 8月土壤含水量 SWC in Aug. (\%) & 高斯模型 Gau & 0.070 & 0.082 & 52 & 0.146 \\
10月土壤含水量 SWC in Oct. (\%) & 高斯模型 Gau & 0.089 & 0.099 & 120 & 0.101 \\
海拔 Altitude & 球状模型 Sph & 0.000 & 0.000 & 140 & 0.855 \\
坡度 Slope & 高斯模型 Gau & 0.021 & 0.131 & 92 & 0.840 \\
坡向 Aspect & 高斯模型 Gau & 0.034 & 0.664 & 150 & 0.949 \\
凹凸度 Convex & 球状模型 Sph & 0.056 & 0.072 & 90 & 0.222 \\
\hline
\end{tabular}

SWC, Soil water content. Exp, Sph, Gau represent the exponential, spherical and Gaussian model, respectively.

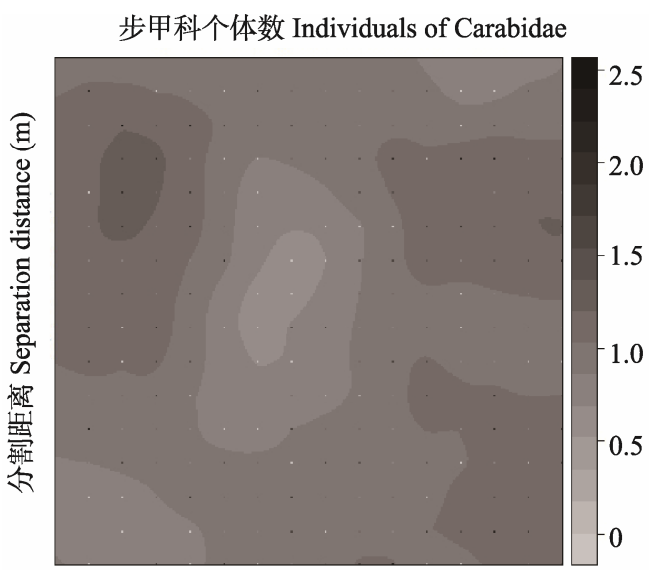

分割距离 Separation distance (m)

隐翅虫科个体数 Individuals of Staphylinidae

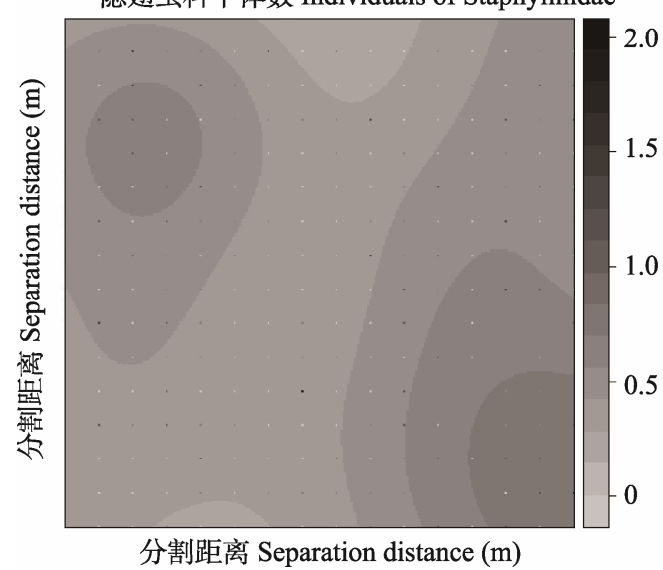

步甲科物种数 Species number of Carabidae

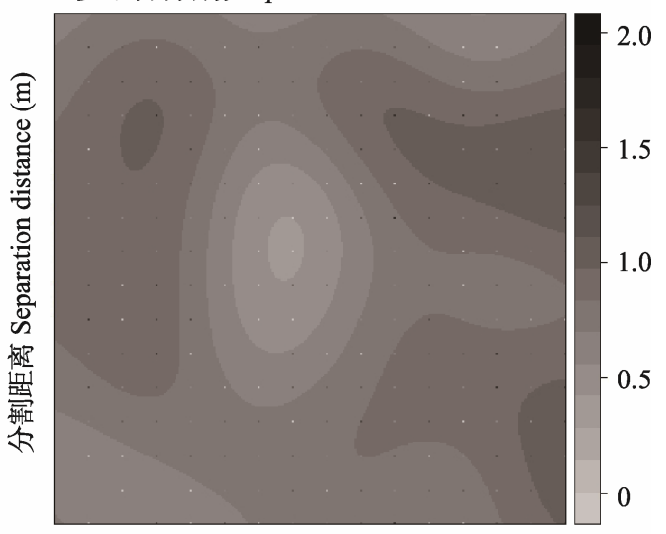

分割距离 Separation distance (m)

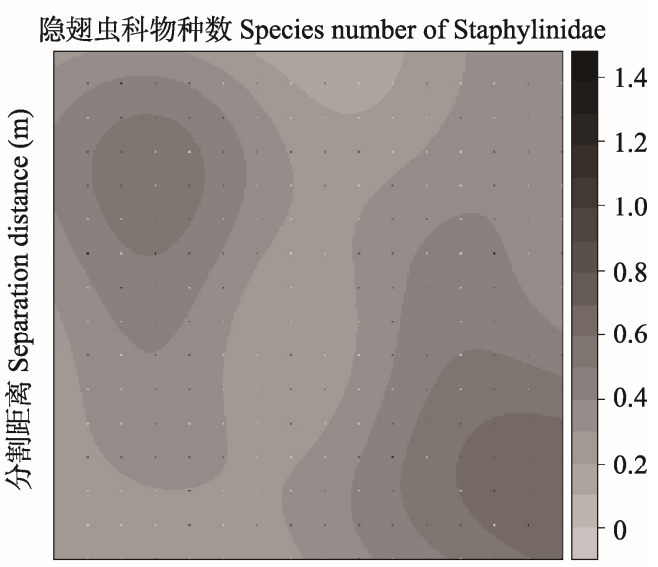

分割距离 Separation distance (m)

图3 8月步甲科和隐翅虫科群落个体数和物种数的空间分布格局

Fig. 3 Spatial patterns for individual and species number matrices of Carabidae and Staphylinidae communities in August

(Megodontus vietinghoffi)分别和Pterostichus maoershanensis 、P. adstrictus、Philonthus wuesthoffi 3 个物 种表现为负的空间关联性。结构比为 0 的物种对有 7 对，其正的或负的空间关联性主要是随机性因素引 起, 有 8 个物种对的结构比大于 0.75 , 其空间关联性 由结构性因素调控。10月Pterostichus adstrictus 和
Platynus ezoanus表现为负的空间关联性，结构比大 于 0.75 (表3)。

\subsection{2 步甲科、隐翅虫科群落个体数与环境因子的} 空间关联性

8 月、 10 月土壤含水量与步甲科、隐翅虫科个 体数在全尺度上表现为负的空间关联性。 


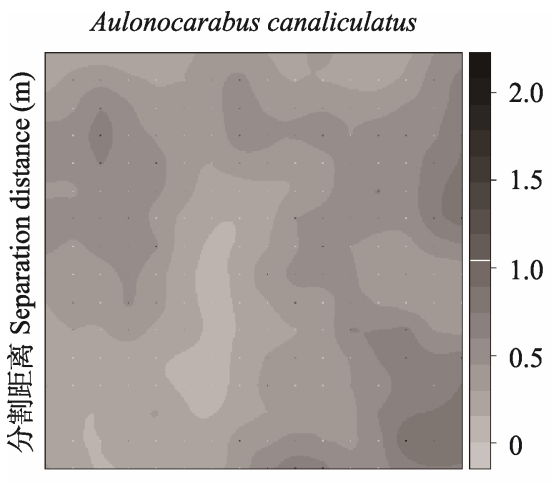

分割距离 Separation distance $(\mathrm{m})$

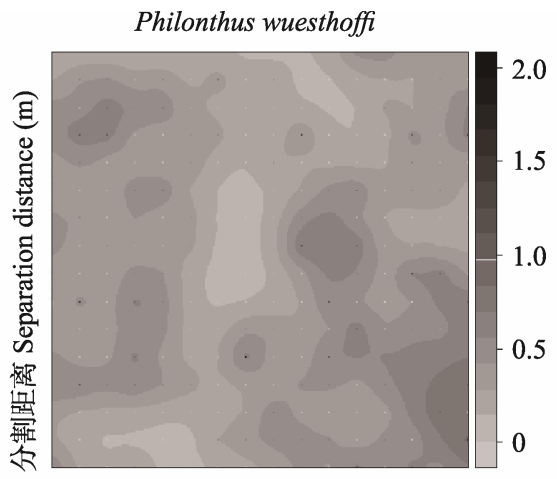

分割距离 Separation distance (m)

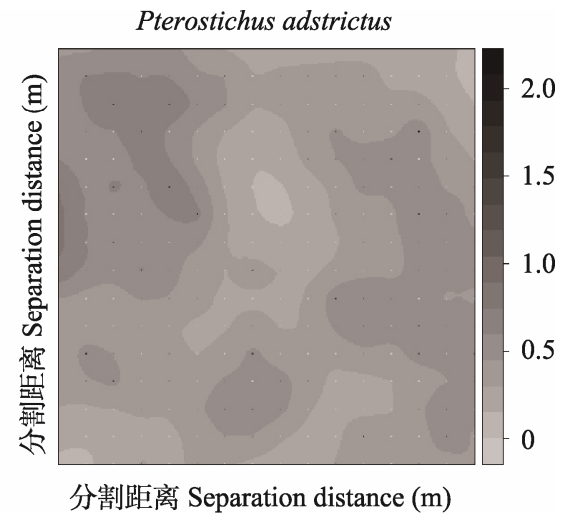

图4 8月优势种个体数的空间分布格局

Fig. 4 Spatial distribution patterns of dominant species individuals in August

表3 8月和10月物种之间的空间关联性

Table 3 Spatial associations between species in August and October

\begin{tabular}{|c|c|c|c|c|}
\hline & $\begin{array}{l}\text { 物种 } \\
\text { Species }\end{array}$ & $\begin{array}{l}\text { 相关关系 } \\
\text { Correlativity }\end{array}$ & $\begin{array}{l}\text { 结构比 } \\
\text { Structural ratio } \\
{\left[C /\left(C_{0}+C\right)\right]}\end{array}$ & $\begin{array}{l}\text { 决定系数 } \\
\text { Coefficient of determina- } \\
\text { tion }\left(R^{2}\right)\end{array}$ \\
\hline \multirow{15}{*}{$\begin{array}{l}\text { 8月 } \\
\text { August } 2015\end{array}$} & Pterostichus maoershanensis - Pterostichus adstrictus & + & 0.000 & 0.116 \\
\hline & Pterostichus maoershanensis - Carabus billbergi & + & 0.565 & 0.325 \\
\hline & Pterostichus maoershanensis - Megodontus vietinghoffi & - & 0.998 & 0.000 \\
\hline & Pterostichus maoershanensis - Aulonocarabus canaliculatus & + & 0.000 & 0.131 \\
\hline & Pterostichus maoershanensis - Philonthus wuesthoffi & + & 0.000 & 0.052 \\
\hline & Pterostichus adstrictus - Carabus billbergi & + & 0.997 & 0.000 \\
\hline & Pterostichus adstrictus - Megodontus vietinghoffi & - & 0.000 & 0.005 \\
\hline & Pterostichus adstrictus - Aulonocarabus canaliculatus & + & 0.959 & 0.383 \\
\hline & Pterostichus adstrictus - Philonthus wuesthoffi & + & 0.998 & 0.174 \\
\hline & Carabus billbergi - Megodontus vietinghoffi & + & 0.000 & 0.000 \\
\hline & Carabus billbergi-Aulonocarabus canaliculatus & + & 0.000 & 0.024 \\
\hline & Carabus billbergi-Philonthus wuesthoffi & + & 0.999 & 0.226 \\
\hline & Megodontus vietinghoffi - Aulonocarabus canaliculatus & + & 0.999 & 0.524 \\
\hline & Megodontus vietinghoffi - Philonthus wuesthoffi & - & 0.000 & 0.247 \\
\hline & Aulonocarabus canaliculatus - Philonthus wuesthoffi & + & 1.000 & 0.532 \\
\hline $\begin{array}{l}\text { 10月 } \\
\text { October } 2015\end{array}$ & Pterostichus adstrictus - Platynus ezoanus & - & 0.999 & 0.000 \\
\hline
\end{tabular}

,+- 分别表示正的和负的空间关联性

,+- represent positive and negative spatial correlations

8 月，海拔与步甲科、隐翅虫科为正的空间关联 性，坡度与步甲科为正的空间关联性，与隐翅虫科 在20-58.34 m之间为负的空间关联性, $58.34 \mathrm{~m}$ 后为 正关联性，坡向与步甲科、隐翅虫科在 $20-85.63 \mathrm{~m}$ 间为空间负关联， $85.63 \mathrm{~m}$ 后为空间正关联，凹凸 度与步甲科为空间正关联, 与隐翅虫科为空间负 关联。

10 月，海拔与步甲科为负的空间关联，但在 $40 \mathrm{~m}$ 范围内存在正的空间关联性, 与隐翅虫科为正
的空间关联，坡度与步甲科在 $100 \mathrm{~m}$ 之后为空间正 关联，与隐翅虫科在全尺度上均是正的空间关联， 坡向与步甲科在 0-20 m、116.69-125.25 m表现为负 的空间关联，与隐翅虫科呈负关联。凹凸度与步甲 科仅在 0-28.82 $\mathrm{m}$ 间为空间正关联, 与隐翅虫科为 正关联(表4)。

\subsection{3 步甲科、隐翅虫科物种与环境因子的相关分析}

$\mathrm{CCA}$ 分析表明, 8月对步甲科群落物种分布影 响最显著的是坡度, 其次是含水量。含水量与第 1 
表4 步甲科和隐翅虫科群落个体数与环境因子空间关联性

Table 4 Spatial associations between individuals of Carabidae and Staphylinidae communities and environmental factors

\begin{tabular}{|c|c|c|c|c|}
\hline \multirow{2}{*}{$\begin{array}{l}\text { 环境因子 } \\
\text { Environmental factor }\end{array}$} & \multicolumn{2}{|l|}{ 8月 August } & \multicolumn{2}{|l|}{ 10月 October } \\
\hline & 步甲科 Carabidae & 隐翅虫科 Staphylinidae & 步甲科 Carabidae & 隐翅虫科 Staphylinidae \\
\hline 土壤含水量 Soil water content & - & - & - & - \\
\hline 海拔 Altitude & + & + & - & + \\
\hline 坡度 Slope & + & + & - & + \\
\hline 坡向 Aspect & + & + & + & - \\
\hline 凹凸度 Convex & + & - & - & + \\
\hline
\end{tabular}

,+- 分别表示正的和负的空间关联性

,+- represent positive and negative spatial correlations

排序轴相关性较大, 海拔与第2排序轴的相关性较 大, Carabus billbergi的分布受坡度和坡向影响较大, 沟步甲(Aulonocarabus canaliculatus)受海拔和含水 量影响较大; 对隐翅虫科群落而言, 含水量对群落 物种分布影响最显著, 含水量与第一排序轴相关性 较大, Philonthus wuesthoffi 受坡度影响较大。10月凹 凸度和海拔对步甲科群落物种分布影响较大。凹凸 度与第 1 排序轴相关性较大, 坡向、含水量与第 2 排 序轴相关性较大, Platynus ezoanus受凹凸度影响较 大; 海拔是对 10 月隐翅虫群落物种分布影响最大的 因子(图5)。

\section{4 讨论}

\section{1 群落物种组成}

研究区步甲科和隐翅虫科成虫群落组成表现 为中等和较强的变异。步甲科和隐翅虫科的群落组 成均具有明显的时间异质性，8月捕获的物种数和 个体数均显著多于 10 月, 可能与步甲科、隐翅虫科 成虫的生物学特性、自身的季节性活动规律以及研 究地气候环境有关。夏季为步甲科、隐翅虫科成虫 活动的高峰期(Lövei \& Sunderland, 1996), 同时试 验区雨热同期, 为其生长、发育和繁殖提供了丰富 的食物资源、多样的栖息环境和适宜的地表温湿度, 使得 8 月群落个体数显著多于 10 月。

\section{2 空间异质性特征}

丰林属于大陆性季风气候, 生长季节和寒冷季 节步甲科、隐翅虫科成虫个体数存在差异(朱纪元等, 2016), 本研究进一步说明生长季节和寒冷季节步 甲科、隐翅虫科成虫空间异质性特征也存在差异。 半方差函数分析结果表明, 10月隐翅虫科物种数和 个体数均表现为较强的空间自相关性, 其空间变异
可能是受到基于生态位理论的环境笁选和种间竞 争的影响(Rusek, 1998)以及隐翅虫科物种的生态学 特征(取食类型、活动时间、活动方式、生长繁殖等) 对影响因子的响应不同(吴东辉等, 2008a)。对于单 个物种种群, 绝大多数物种具有中等的空间自相关 性，且均存在块金值，即存在由实验误差及小于采 样尺度和微尺度的空间变异带来的块金效应。陷阱 法反映的并非物种的真实密度而是物种的活动性 密度(吴东辉等, 2008b; 祝向钰等, 2012), 且步甲 科、隐翅虫科成虫的活动密度与土壤含水量、植物 生物量、植物盖度和植被类型密切相关(Christiansen \& Bellinger, 1980; 陈建秀等, 2007)。因此小于采样 尺度 $(20 \mathrm{~m})$ 的空间过程不容忽视，同时物种个体数 量稀少或频度较低(空间聚集分布或样圆数目和大 小引起)也是导致难以揭示其空间相关性的一个原 因。若要揭示其空间分异规律, 需要在现有采样幅 度内加大采样密度、缩小空间粒度进行对比分析(史 文娇等, 2012)。土壤含水量和凹凸度空间自相关性 较弱, 其中 8 月土壤含水量的空间自相关距离明显 较短 $(52 \mathrm{~m})$, 而其他环境因子均具有较强的空间自 相关性，空间自相关距离相对较长(>92 m)。

\section{3 分布格局}

克里格插值结果表明物种数和个体数分布格 局相似，步甲科群落分布格局与隐翅虫科格局相 似。这可能是由于聚集地区食物资源较丰富，能满 足不同物种的需求，也有可能是步甲科与隐翅虫科 之间存在某种依赖协作行为。10月物种个体数较少, 空间格局单一，斑块结构不明显，可能与微域地 形、植被斑块、食物资源梯度(赵雪等, 2015)或者地 面植被位置、生物繁殖和竞争等(徐丽娜和金光泽, 2012)有关。从夏季到寒冷季节，群落分布格局的变 

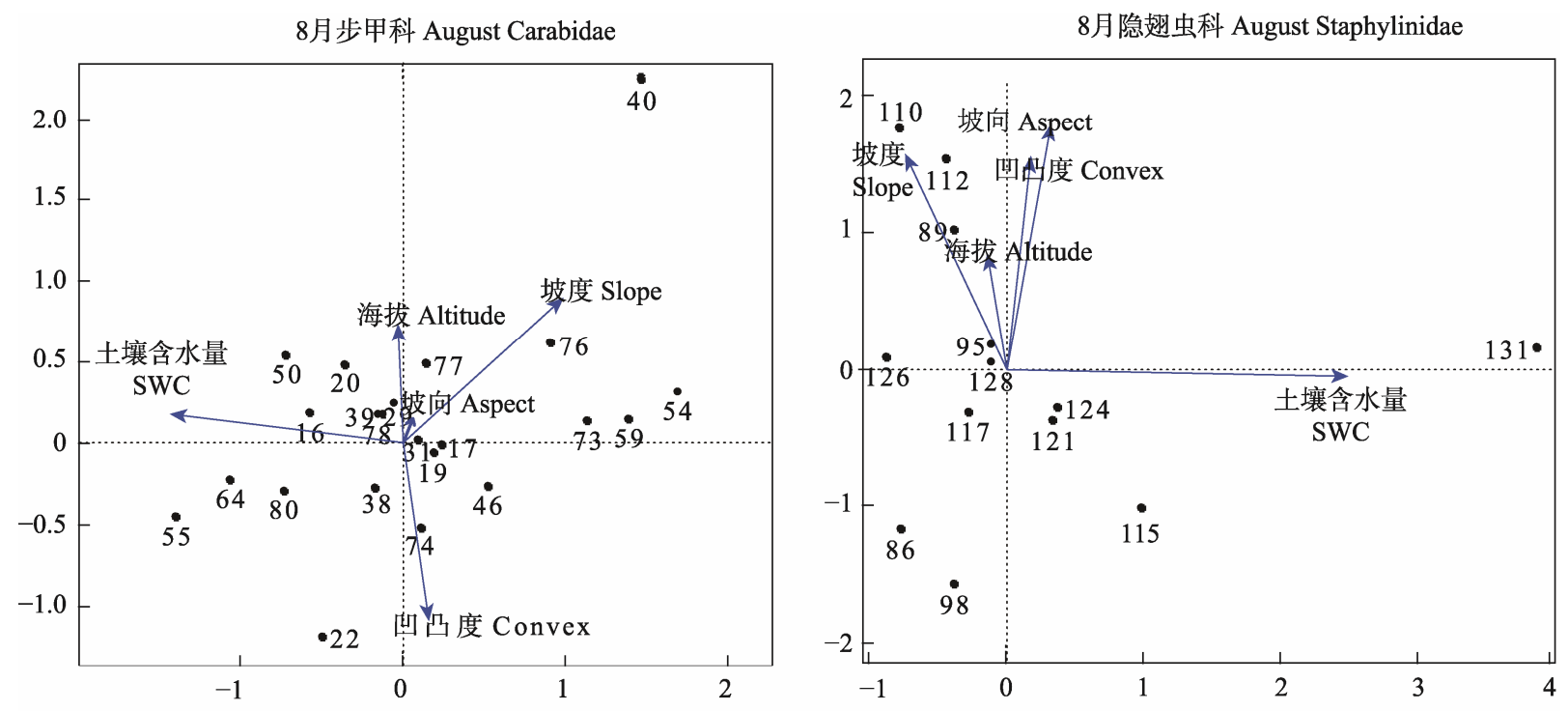

10月步甲科 October Carabidae

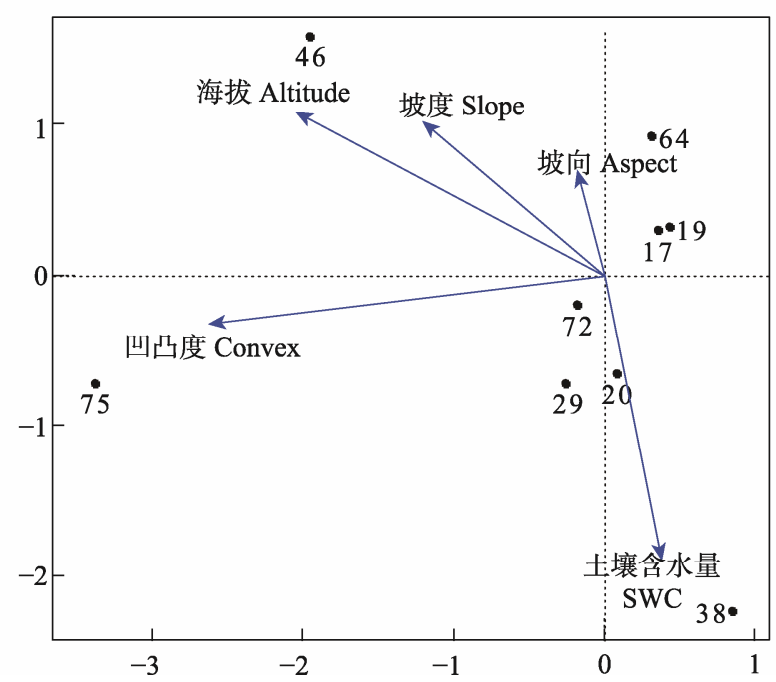

10月隐翅虫科 October Staphylinidae

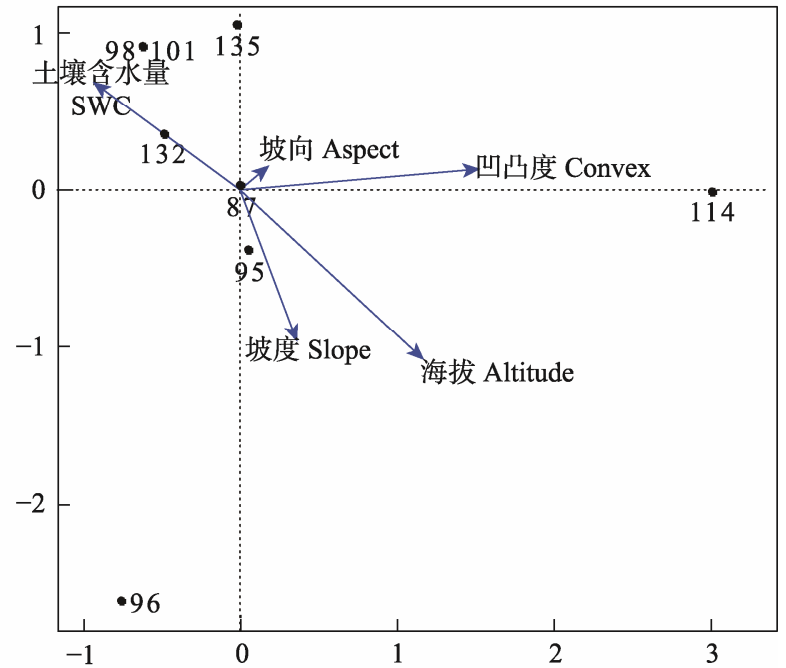

图5 步甲科、隐翅虫科物种个体数与环境因素的典范对应分析二维排序图

Fig. 5 Biplot of individuals for Carabidae and Staphylinidae to environmental variables

16, Pterostichus (Metallophilus) heilongjiangensis; 17, Pterostichus maoershanensis; 19, Pterostichus adstrictus; 20, Pterostichus bituberculatus; 22, Pterostichus microcephalus; 23, Pterostichus orientalis; 29, Pterostichus sulcitarsis; 31, Carabus billbergi; 38, 金边步甲(Megodontus vietinghoffi); 39, 沟步甲(Aulonocarabus canaliculatus); 40, Nebria livida; 46, Anthracus horni; 50, Bembidion lissontum; 54, Bembidion pseadducillum; 55, Biphyllidae cryptophilus obliterates; 59, Colpodes elainus; 64, Dicranoncus femoralis; 72, Platynus ezoanus; 73, Platynus gracilis; 74, Platynus ogurae; 75, Platynus thoreyi; 76, Pristosia proxima; 77, Pristosia vigil; 78, Stenolophus difficilis; 79, Syncechus callitheres; 80, Syncechus cycloderus; 86, Acidota chinensis; 87, Acotylus mimulus; 89, Aleochara curtula; 95, Anotylus minulus; 96, Boreaphilus japonicas; 98, Carpelimus vagus; 101, Boreaphilus japonicas; 110, Philonthus aeneipenuis; 111, Philonthus brannicollis; 112, Philonthus cyanipennis; 114, Philonthus gastralis; 115, Philonthus havellkai; 117, Philonthus numata; 121, Philonthus sericans; 124, Philonthus solidus; 126, Philonthus tenuicornis; 128, Philonthus wuesthoffi; 131, Psephidonus sinuatus; 132, Qachyporus celatus; 135, Tachyporus celatus. SWC, Soil water content.

化一方面可能与土壤含水量、地表温湿度以及调落 物厚度等非确定性因素具季节性变化有关, 另一方 面可能与步甲科、隐翅虫科成虫自身的生物学特性 (如食物资源) 有关, 也可能是 10 月温度条件相对较 低，导致大量甲虫死亡，使得所捕获物种整体较
少，物种活动的范围扩大导致物种分布零散。环境 因子的克里格插值分析中, 坡度的分布格局表现 为中间低值，边缘高值，与步甲科和隐翅虫科分 布格局相似, 即在坡度较大的区域, 物种分布较 多。土壤含水量 8 月和10月的分布格局相似，在样 
地边缘含水量较低, 而物种在边缘较为集中(数据 未列出)。虽然相关研究表明步甲科和隐翅虫科均 喜好潮湿的生境, 本实验并未在土壤含水量较大 的地区发现较多的物种聚集现象, 实际上步甲科 和隐翅虫科个体数与土壤含水量呈现负的空间关 联性(见表4)，这暗示这些甲虫的空间分异可能同 时受到其他因子的影响。甲虫的生物学特性以及周 围的生活环境决定了步甲科和隐翅虫科有不同的 空间分布型。

\section{4 空间关联性}

交叉方差函数分析结果表明步甲科和隐翅虫 科物种间多为正的空间关联性, 物种之间的正的或 负的空间关联性主要受随机性因素或结构性因素 单一控制, 简单Mantel检验进一步表明物种间的空 间关联性并不显著, 说明这些物种间存在着伪的 (spurious)空间关联性，即这些空间关联性可能由简 单的空间格局形成(Gutiérrez-López et al, 2010)。分 析环境因子(土壤含水量、地形)与群落的空间关联 性发现, 物种个体数与环境因子的空间关联性在不 同尺度上表现不同, 但多数发生在样点的小范围内 或所监测范围最大值处(数据未展示), 且不同月份 之间相同因子的空间关联性也有所变化, 因此地形 因子可能不是步甲科、隐翅虫科群落分布格局的主 导因子。通过CCA分析物种与环境因子之间的相关 程度发现 8 月含水量对步甲科和隐翅虫科物种分布 影响较大, 其次是坡度, 海拔对物种的分布影响较 小，10月海拔和凹凸度对物种分布影响较大。本实 验所测的环境因子较少 (土壤含水量、地形因子), 很 难代表全部重要的环境因子, 且本文使用的陷阱法 主要反映的是地表鞘翅目成虫活动密度的空间异 质性特征, 为了揭示环境和步甲科、隐翅虫科物种 之间的复杂空间作用关系, 需要在以后的实验中同 时考虑其他的环境因子(如地表植被因子、地表调落 物特征和微气候因子), 并进一步改进野外调查方 法，在不同时空尺度上开展综合探讨。

致谢：感谢李景科老师在物种鉴定中的帮助。感谢 朱纪元、间龙、曹阳、胡媛媛在野外调查和物种鉴 定中的帮助。感谢夏尚文在数据分析中提供的帮 助。感谢丰林自然保护区在实验开展过程中提供的 支持。

\section{参考文献}

Bivand RS, Pebesma EJ, Gómezrubio V (2013) Applied Spatial Data Analysis with R. Springer Science and Business Media, New York.

Cambardella CA, Moorman TB, Karlen DL, Novak JM, Turco RF, Konopka AE (1994) Field-scale variability of soil properties in central Iowa soils. Soil Science Society of America Journal, 58, 1501-1511.

Chen JX, Ma ZC, Yan HJ, Zhang F (2007) Roles of springtails in soil ecosystem. Biodiversity Science, 15, 154-161. (in Chinese with English abstract) [陈建秀, 麻智春, 严海娟, 张峰 (2007) 跳虫在土壤生态系统中的作用. 生物多样 性, 15, 154-161.]

Christiansen K, Bellinger PF (1980) Collembola of North America, North of the Rio Grand. Grinnell College, Grinnell Iowa.

Fernandez-Going BM, Adler PB, D’Antonio C (2014) How Does Spatial Heterogeneity Buffer Plant Species Against Climate Change? ESA Convention.

Gao MX, Liu D, Zhang XP, Wu DH (2016) Spatial relationships between the abundance of aboveground and belowground soil mite communities, and environmental factors in farmland on the Sanjiang Plain, China. Acta Ecologica Sinica, 36, 1782-1792. (in Chinese with English abstract) [高梅香, 刘冬, 张雪萍, 吴东辉 (2016) 三江平原农田地 表和地下土壤螨类丰富度与环境因子的空间关联性. 生 态学报, 36, 1782-1792.]

Guo XD, Fu BJ, Ma KM, Chen LD, Yang FL (2000) Spatial variability of soil nutrients based on geostatistics combined with GIS: A case study in Zunhua City of Hebei Province. Chinese Journal of Applied Ecology, 11, 557-563. (in Chinese with English abstract) [郭旭东, 傅伯杰, 马克明, 陈 利顶, 杨福林 (2000) 基于GIS和地统计学的土壤养分空 间变异特征研究一以河北省遵化市为例. 应用生态学报, 11, 557-563.]

Gutiérrez-López M, Jesús JB, Trigo D, Fernández R, Novo M, Díazi-Cosín DJ (2010) Relationships among spatial distribution of soil microarthropods, earthworm species and soil properties. Pedobiologia, 53, 381-389.

Holt RD (1984) Spatial heterogeneity, indirect interactions, and the coexistence of prey species. The American Naturalist, 124, 377-406.

Jia YH, Zhao CY, Nan ZR, Wan YX (2008) Spatial heterogeneity of soil salinity in groundwater-fluctuating region of the lower reaches of the Heihe River. Acta Pedologica Sinica, 45, 420-430. (in Chinese with English abstract) [贾艳红, 赵传燕, 南忠仁, 万研新 (2008) 黑河下游地下水波动带 土壤盐分空间变异性研究. 土壤学报, 45, 420-430.]

Lei ZD, Yang SX, Xie SZ (1998) Soil Water Dynamics. Tsinghua University Press, Beijing. (in Chinese) [雷志栋, 
杨诗秀, 谢森传 (1988) 土壤水动力学. 清华大学出版 社, 北京.]

Li HR, Reynolds JF (1995) On definition and quantification of heterogeneity. Oikos, 73, 280-284.

Li JK, Lin L, Zhang XP (2015a) Primary Colour Illustration of Soil Beetles in Northeastern China (Carabidae). Harbin Map Press, Harbin. (in Chinese) [李井科, 林琳, 张雪萍 (2015a) 原色中国东北土壤甲虫图鉴(步行虫类). 哈尔滨 地图出版社，哈尔滨.]

Li JK, Zhang LM, Zhang XP (2015b) Primary Colour Illustration of Soil Beetles in Northeastern China (Staphylinidae and Tenebrionidae). Harbin Map Press, Harbin. (in Chinese) [李井科, 张利敏, 张雪萍 (2015b) 原色中国东北土壤甲 虫图鉴(隐翅虫类, 拟步甲类). 哈尔滨地图出版社, 哈尔 滨.]

Li JQ, Li JW (2003) Regeneration and restoration of broad-leaved Korean pine forests in Lesser Xing'an Ridge in mountains of northeast China. Acta Ecologica Sinica, 23, 1268-1277. (in Chinese with English abstract) [李俊清, 李 景文 (2003) 中国东北小兴安岭阔叶红松林更新及其恢 复研究. 生态学报, 23, 1268-1277.]

Li YS, Wang GX, Ding YJ, Wang YB, Zhao L, Zhang CM (2008) Spatial heterogeneity of soil moisture in alpine meadow area of the Qinghai-Xizang Plateau. Advances in Water Science, 19, 61-67. (in Chinese with English abstract) [李元寿, 王根绪, 丁永建, 王一博, 赵林, 张春敏 (2008) 青藏高原高寒草甸区土壤水分的空间异质性. 水 科学进展, 19, 61-67.]

Liu LX, Liu CZ, Mao ZJ (2011) Evaluation of forest ecosystem service functions in Fenglin Biosphere Nature Reserve, Heilongjiang Province. Journal of Beijing Forestry University, 33(3), 38-44. (in Chinese with English abstract) [刘林 馨, 刘传照, 毛子军 (2011) 丰林世界生物圈自然保护区 森林生态系统服务功能价值评估. 北京林业大学学报, 33(3), 38-44.]

Lövei GL, Sunderland KD (1996) Ecology and behavior of ground beetles (Coleoptera: Carabidae). Annual Review of Entomology, 41, 231-256.

Questad EJ, Foster BL (2008) Coexistence through spatio-temporal heterogeneity and species sorting in grassland plant communities. Ecology Letters, 11, 717-726.

Rainio J, Niemelä J (2003) Ground beetles (Coleoptera: Carabidae) as bioindicators. Biodiversity and Conservation, $12,487-506$

Rossi JP, Lavelle P, Tondoh JE (1995) Statistical tool for soil biology. X. Geostatistical analysis. European Journal of Soil Biology, 31(4), 173-181.

Rusek J (1998) Biodiversity of Collembola and their functional role in the ecosystem. Biodiversity and Conservation, 7, 1207-1219.

Shi WJ, Yue TX, Shi XL, Song W (2012) Research progress in soil property interpolators and their accuracy. Journal of Natural Resources, 27, 163-175. (in Chinese with English abstract) [史文娇, 岳天祥, 石晓丽, 宋伟 (2012) 土壤连 续属性空间插值方法及其精度的研究进展. 自然资源学 报, 27, 163-175.]

Sousa JP, Gama MMD, Ferreira CS (2003) Effects of replacing oak-woods by eucalyptus on edaphic Collembola communities: Does the size and type of plantation matter. Acta Entomologica Ibérica e Macaronésica, 1, 1-10.

Wang ZJ, Li DM, Shang HW, Cheng JA (2002) Theories and methods of geostatistics and their application in insect ecology. Entomological Knowledge, 39, 405-411. (in Chinese with English abstract) [王正军, 李典谟, 商晗武, 程家安 (2002) 地质统计学理论与方法及其在昆虫生态学中的应 用. 昆虫知识, 39, 405-411.]

Wang ZQ (1999) Geostatistics and Its Application in Ecology. Science Press, Beijing. (in Chinese) [王政权 (1999) 地统 计学及在生态学中的应用. 科学出版社, 北京.]

Wu DH, Yin WY, Yan RQ (2008a) Influence of vegetation reclamation type on the characteristics of soil collembola community in seriously alkalinized and degraded grasslands of Songnen Plain. China Environmental Science, 28, 466-470. (in Chinese with English abstract) [ 吴东辉, 尹文 英, 阎日青 (2008a) 植被恢复方式对重度退化草原土壤 跳虫群落的影响. 中国环境科学, 28, 466-470.]

Wu DH, Yin WY, Yin XQ (2008b) Comparisons among soil collembola community characteristics in relation to different vegetation restoration treatments in the moderate degraded grasslands in the Songnen Plain of Northeast China. Acta Entomologica Sinica, 51, 509-515. (in Chinese with English abstract) [吴东辉，尹文英，殷秀琴 (2008b) 松嫩草原中 度退化草地不同植被恢复方式下土壤跳虫群落特征比较。 昆虫学报, 51, 509-515.]

Wu QB, Shen YP, Shi B (2003) Relationship between frozen soil together with its water-heat process and ecological environment in the Tibetan Plateau. Journal of Glaciology and Geocryology, 25, 250-255. (in Chinese with English abstract) [吴青柏, 沈永平, 施斌 (2003) 青藏高原冻土及水 热过程与寒区生态环境的关系. 冰川冻土, 25, 250-255.]

Xia SW, Chen J, Schaefer D, Goodale UM (2016) Effect of topography and litterfall input on fine-scale patch consistency of soil chemical properties in a tropical rainforest. Plant and Soil, 404, 385-398.

Xu LN, Jin GZ (2012) Species composition and community structure of a typical mixed broad-leaved Korean pine (Pinus koraiensis) forest plot in Liangshui Nature Reserve, Northeast China. Biodiversity Science, 20, 470-481. (in Chinese with English abstract) [徐丽娜, 金光泽 (2012) 小 兴安岭凉水典型阔叶红松林动态监测样地: 物种组成与 群落结构. 生物多样性, 20, 470-481.]

Yin WY (1997) Ten-year progress in the study of soil zoology 
in China. China Science Foundation, 11, 48-51. (in Chinese) [尹文英 (1997) 中国土壤动物学研究10年进展. 中国科 学基金, 11, 48-51.]

Zhao X, Xu LN, Jin GZ (2015) Effects of topography on shrub regeneration in typical broad-leaved Korean pine forests. Biodiversity Science, 23, 767-774. (in Chinese with English abstract) [赵雪, 徐丽娜, 金光泽 (2015) 地形对典型阔叶 红松林灌木更新的影响. 生物多样性, 23, 767-774.]

Zhou HZ, Yu XD, Luo TH, Li XY, Wang FY, Li DE, Zhou YLZ, Zhao CY (2014) Collection methods and sampling technique of ground dwelling and predating Carabids and Staphylinids beetles. Journal of Applied Entomology, 51, 1367-1375. (in Chinese with English abstract) [周红章, 于 晓东, 罗天宏, 李晓燕, 王凤艳, 李德娥, 周毓灵子, 赵 彩云 (2014) 土壤步甲和隐翅虫的采集与田间调查取样 技术. 应用昆虫学报, 51, 1367-1375.]
Zhu JY, Li JK, Cheng SS, Yan L, Hu YY, Cao Y, Ni JP, Liu J, Wang JF, Gao MX (2016) Community structure of adult Coleoptera on local scale in a mixed broad-leaved Korean pine forest in the Xiao xing'an Mountains. Journal of Northeast Forestry University, 44(12), 57-63. (in Chinese with English abstract) [朱纪元，李景科，程赛赛，间龙，胡 媛媛，曹阳，倪娟平，刘俊，王继富，高梅香 (2016) 小 兴安岭阔叶红松林局地尺度地表鞘翅目成虫群落结构. 东北林业大学学报, 44(12), 57-63.]

Zhu XY, Li ZY, Chang L (2012) Changes in composition and the quantity of $B t$ in rice soil Collembola community. Acta Ecologica Sinica, 32, 3546-3554. (in Chinese with English abstract) [祝向钰, 李志毅, 常亮 (2012) 转 $B t$ 水稻土壤跳 虫群落组成及其数量变化. 生态学报, 32, 3546-3554.]

(责任编委：吴东辉 责任编辑：问文杰) 